Ambiente \& Água - An Interdisciplinary Journal of Applied Science
ISSN 1980-993X - doi:10.4136/1980-993X
www.ambi-agua.net
E-mail: ambi.agua@gmail.com

\title{
Evaluación de cambio de cobertura vegetal y uso de suelo en la cuenca del río Tecolutla, Veracruz, México; periodo 1994-2010
}

\author{
doi: 10.4136/ambi-agua.1539
}

Received: 13 Oct. 2014; Accepted: 22 Jan. 2015

\author{
Ana Karen Osuna-Osuna; José De Jesús Díaz-Torres; José De Anda-Sánchez; \\ Edgardo Villegas-García; Juan Gallardo-Valdez; Gustavo Davila-Vazquez* \\ Centro de Investigación y Asistencia en Tecnología y Diseño del Estado de Jalisco, A. C., \\ Guadalajara, Jalisco, México \\ Unidad de Tecnología Ambiental \\ *Autor correspondiente: e-mail: gdv@ ciatej.mx, \\ osuna_anakaren@hotmail.com,jdiaz@ciatej.mx,janda@ciatej.mx, \\ evillegas@ciatej.mx,jgallardo@ciatej.mx
}

\section{RESUMEN}

Los estudios relacionados con los cambios progresivos en la cobertura vegetal y de uso de suelo, han cobrado importancia en la investigación ambiental, ya que han permitido evaluar las tendencias espacio-temporales de procesos como la deforestación y degradación ambiental, provocadas por actividades humanas. En este contexto, el objetivo de este estudio fue evaluar el impacto de las actividades antropogénicas en la cuenca del río Tecolutla, Veracruz, México; en un periodo de 16 años. Se utilizaron imágenes de satélite Landsat para los años de 1994 y 2010, distinguiéndose nueve usos y coberturas del suelo: selva, bosques, suelo agrícola, agua, vegetación perturbada, asentamientos urbanos, pastizales, cultivos de cítricos y matorrales. Se validaron los mapas temáticos, obteniéndose exactitudes globales mayores a 92\%, y coeficientes de Kappa de 0.89 y 0.91 para las clasificaciones de 1994 y 2010, respectivamente. El análisis de la matriz de transición reveló una tendencia al incremento de superficies dedicadas a actividades humanas (agricultura y uso urbano), presentando porcentajes de cambio de $28 \%$ y $67 \%$ en un lapso de 16 años, respectivamente. En consecuencia, se observó una disminución ( $-1.1 \%$ anual) de superficies con coberturas naturales, específicamente de bosques y selvas, encontrándose similitudes con trabajos realizados a nivel nacional y estatal, donde se ha demostrado la transición de coberturas naturales por el incremento de actividades antropogénicas. Los resultados de este trabajo son de utilidad para futuros planes de desarrollo ambiental, planes de ordenamiento territorial, y en la planeación de estrategias de conservación de los recursos naturales en la cuenca.

Palabras clave: clasificación de máxima verosimilitud, coeficiente de Kappa, Landsat TM, matriz de transición, pérdida de la biodiversidad.

\section{Avaliação da mudança da vegetação e uso do solo na bacia do rio Tecolutla, Veracruz, México; no período de 1994-2010}

\section{RESUMO}

Estudos relacionados a mudanças na vegetação e no uso do solo ganharam importância em pesquisas ambientais, o que tem permitido conhecer as tendências espaço-temporal de 
fenômenos como o desmatamento e degradação do meio ambiente, causada principalmente por atividades humanas. Neste contexto, o principal objetivo deste estudo foi avaliar os impactos das atividades humanas na bacia do rio Tecolutla, Veracruz, México, em um período de 16 anos. Imagens do satélite Landsat dos anos de 1994 e 2010 foram utilizadas, distinguindo-se as classes de solo: floresta, bosques, terras agrícolas, água, vegetação perturbada, assentamentos urbanos, pastagens, culturas dos citros e arbustos. Os mapas temáticos foram validados e resultarem em precisão global superior a $92 \%$ e os coeficientes kappa de 0,89 e 0,91 para as classificações de 1994 e 2010, respectivamente. A análise da matriz de transição revelou uma tendência de aumentar as áreas sob as atividades humanas (agricultura e uso urbano), com aumento de $28 \%$ e $67 \%$ em 16 anos, respectivamente. Consequentemente, uma diminuição $(-1,1 \%$ ao ano) de superfícies naturais, principalmente de matas e florestas. Esses resultados estão consistentes com valores reportados em nível nacional e estadual, que têm mostrado a transição no uso do solo devido a ações antropogênicas. Os resultados deste estudo poderão ser úteis para planos futuros de desenvolvimento ambiental, de uso da terra e para o planejamento de estratégias para a conservação dos recursos naturais na bacia.

Palavras-chave: classificação de máxima verossimilhança, coeficiente Kappa, Landsat TM, matriz de transição, perda de biodiversidade.

\title{
Assessment of land use and land cover change in Tecolutla River Basin, Veracruz, Mexico; during the period 1994-2010
}

\begin{abstract}
Studies related to changes in vegetation and land use cover have gained importance in environmental research, as they allow for the assessment of time-space trends in deforestation and environmental degradation processes, especially as caused by human activity. In this context, the main goal of this study was to evaluate the impact of human activity in the basin of the Tecolutla River, in Veracruz, Mexico during a 16-year period. Landsat satellite images were used for the years 1994 and 2010, distinguishing nine land use coverage classes: rainforest, forest, agricultural land, water, disturbed vegetation, urban settlements, grasslands, citrus crops and shrubs. Thematic maps were validated, yielding overall accuracies greater than $92 \%$ and Kappa coefficients of 0.89 and 0.91 for the 1994 and 2010 classifications, respectively. Analysis of the transition matrix revealed a trend of increasing areas related to human activity (agriculture and urban use) showing percentage changes of $28 \%$ and $67 \%$ within 16 years, respectively. Consequently, a decrease (-1.1\% per year) in areas with natural cover, specifically forest and jungle, was observed. Similar findings were reported in works done at national and state levels, where the transition of natural cover by the increasing of anthropogenic activities has been proven. The results of this study are useful for future environmental development planning, land management planning and planning strategies for the conservation of the natural resources in the basin.
\end{abstract}

Keywords: kappa coefficient, Landsat TM, loss of biodiversity, maximum likelihood classification, transition matrix.

\section{INTRODUCCIÓN}

Los procesos relacionados con el cambio de uso de suelo y pérdida de cobertura vegetal han atraído la atención de un amplio grupo de investigadores en las últimas décadas, desde los dedicados a la modelación de patrones espacio-temporales de la conversión de uso de suelo, 
hasta aquellos interesados en la comprensión de las causas y consecuencias de estos cambios (Long et al., 2007).

Durante este proceso de cambio, es común que ocurran una serie de fenómenos naturales, tales como huracanes e incendios, que afecten de manera importante en la pérdida de cobertura vegetal. Sin embargo, en el último siglo y particularmente durante las últimas décadas, han sido las actividades humanas las que han desencadenado en mayor grado estos efectos, como consecuencia de factores demográficos y económicos (Berlanga et al., 2010; Loya-Carrillo et al., 2013).

En México, el cambio en la cobertura vegetal y de uso de suelo se ha presentado desde la época precolombina, no obstante, en los últimos 50 años este proceso ha incrementado de forma alarmante (Ibarra-Montoya et al., 2011). Según la Organización de las Naciones Unidas para la Agricultura y la Alimentación (FAO), las 70 millones de hectáreas de bosques con las que contaba el país en el año 1990, han presentado una tasa de deforestación promedio de 354 mil ha/año hasta el año 2010 (FAO, 2010).

En el estado de Veracruz, México, el sector agropecuario desempeña un papel importante dentro de la actividad económica y la producción de alimentos. Este crecimiento ha tenido un efecto directo en la reducción de la vegetación natural, a medida que se han incrementado las áreas de cultivo y de pastizales inducidos (Hernández et al., 2013; Ellis et al., 2011; Toledo y Ordoñez, 1998).

La cuenca del río Tecolutla, cuya superficie comprende parte de los estados de Veracruz, Puebla, Tlaxcala e Hidalgo, ha manifestado un fuerte impacto sobre sus recursos naturales debido a los procesos de cambio en el uso de suelo. Las actividades agrícolas, las cuales han incrementado de forma alarmante durante los últimos años, representan uno de los principales factores por los cuales han ocurrido cambios en esta región. En el año 2003 se reportaron 70,634 ha cultivadas en los municipios de la cuenca (SIAP, 2012a), mientras que para el 2010, la superficie se incrementó a 96,160 ha (SIAP, 2012a), lo que representa un incremento de $36 \%$ en un periodo de sólo 7 años. Estos cambios han venido modificando la distribución espacial de la cobertura vegetal dentro de la cuenca.

Mediante el incremento significativo de la calidad de los datos de teledetección y la utilización de Sistemas de Información Geográfica (SIG), es posible conocer la dinámica de los cambios de uso de suelo en diferentes periodos, además de entender cómo y por qué ocurren estos cambios; inclusive permite identificar las áreas más susceptibles a presentar cambios, de modo que es fundamental en la toma de decisiones para la planificación de un territorio (Dzieszko, 2014). Ejemplos representativos de la aplicación de este tipo de técnicas en los que se han evaluado cambios de cobertura vegetal y uso de suelo se pueden encontrar en estudios realizados por Alexakis et al. (2014), Berlanga et al. (2010), Loya-Carrillo et al. (2013) y Rodríguez-Galeano et al. (2010).

Bajo esta perspectiva, el presente estudio tiene por objetivo evaluar el impacto de las actividades antropogénicas en la cuenca del río Tecolutla, Veracruz, México; en un periodo de 16 años, por ser considerada como una de las 110 regiones hidrológicas prioritarias identificadas por la Comisión Nacional de la Biodiversidad (CONABIO) en México, debido a su alta biodiversidad (Pérez-Maqueo et al., 2011). Esta investigación cobra importancia al establecer un precedente para identificar y cuantificar las coberturas vegetales que han presentado mayor porcentaje de afectación en esta región, así como para conocer los usos de suelo que las han propiciado. Esta información será de utilidad para futuros planes de desarrollo ambiental y futuras estrategias de conservación de los recursos hídricos de la cuenca, puesto que este tipo de estudios son escasos en México. 


\section{1. Área de estudio}

La cuenca del río Tecolutla pertenece a la región hidrológica $\mathrm{N}^{\circ} 27$ (RH-27) TuxpanNautla. Comprende parte de los estados de Puebla, Veracruz, Tlaxcala e Hidalgo entre las coordenadas UTM: 2153727 y 2266992 de latitud norte y 578205 y 713440 de longitud oeste (Figura 1). Su extensión territorial es de aproximadamente $7,446 \mathrm{~km}^{2}$ (CONAGUA, 2012).

En esta región se identifican cuatro tipos diferentes de climas, de acuerdo a la clasificación de Wladimir Köppen, modificada por Enriqueta García en 1964 (Trejo, 2000): 1) cálido subhúmedo, que predomina en la parte baja de la cuenca cubriendo la llanura costera hasta los 1,000 metros sobre el nivel del mar (msnm); 2) semicálido húmedo, de los 1,000 a 1,6000 msnm, 3) climas templados con abundantes lluvias en verano, desde los 1,600 hasta los 2,800 msnm y 4) clima semifrío subhúmedo, en la parte alta de la cuenca. La temperatura media anual varía de 12 a $24^{\circ} \mathrm{C}$ y la precipitación total anual de 1,200 hasta más de 4,000 mm, siendo esta región la más lluviosa del estado (INEGI, 2005).

La heterogeneidad de la cuenca, relacionada con los contrastes topográficos, características climáticas y edafológicas, ha generado una alta diversidad de hábitats, sin embargo, muchos de estos hábitats manifiestan diversos grados de afectación a lo largo de la cuenca. En las partes altas (estados de Puebla, Tlaxcala e Hidalgo), se presenta la mayor biodiversidad, encontrándose bosques de coníferas, encino y mesófilo de montaña (Pérez.Sesma, 2009). En la parte baja (estado de Veracruz), la biodiversidad es escasa, predominando zonas agrícolas, pastizal inducido y en menor proporción selva subcaducifolia (INEGI, 2007; Pérez-Sesma, 2009).

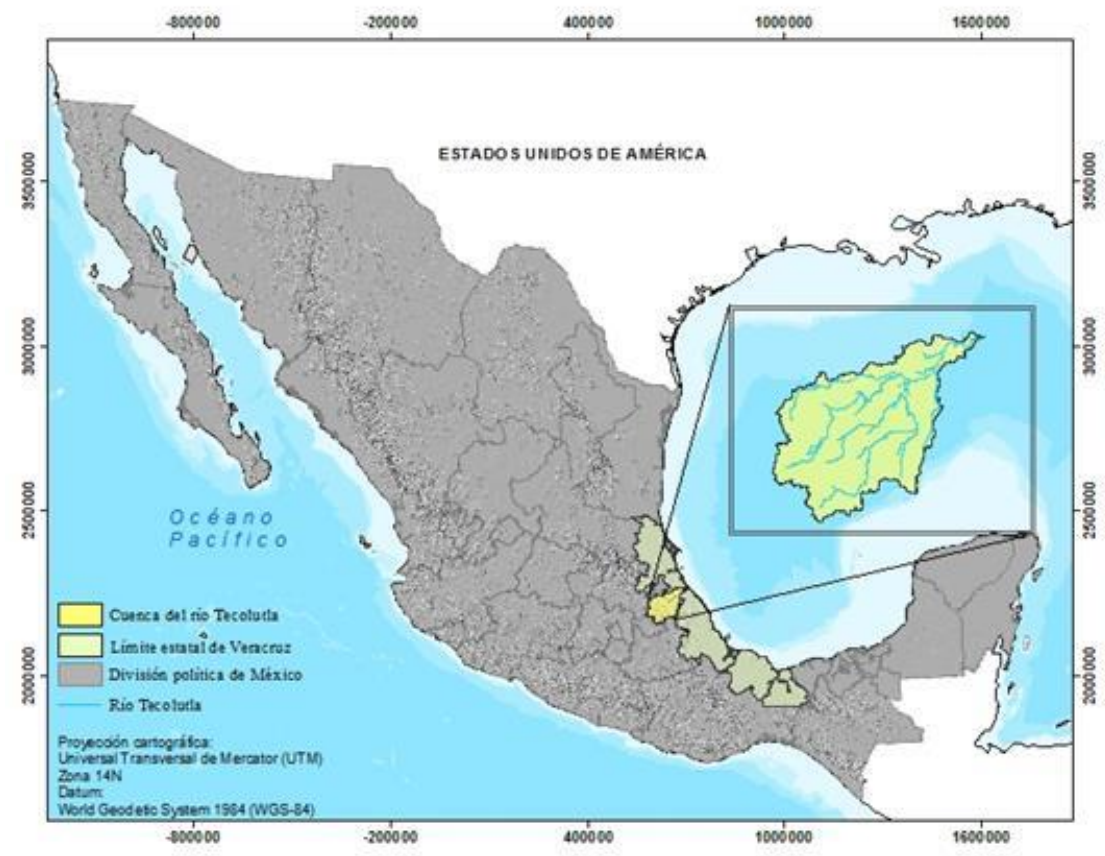

Figura 1. Ubicación de la cuenca del río Tecolutla.

El tipo de agricultura en los municipios que integran la cuenca es de temporal, la cual se realiza principalmente en áreas semiplanas y lomeríos. Durante el año 2012, se cultivaron 95,652 ha; el maíz fue el cultivo predominante (50.3\%), seguido por la naranja (33.5\%) y otros cítricos $(5.2 \%)$ tales como el limón, toronja, mandarina, tangelo y tangerina. En lo que respecta a la producción, la naranja ocupó el primer lugar con una producción de 336,810 toneladas $(55.7 \%)$ seguida por el maíz con 81,789 toneladas (13.5\%), limón con 39,296 toneladas $(6.5 \%)$, jícama con 37,530 toneladas $(6.2 \%)$ y plátano con 35,135 toneladas $(5.8 \%)$ (SIAP, 2012a). 
La actividad ganadera también ha contribuido a la eliminación de la vegetación natural al ser desmontada para la introducción de diversos pastizales. Las principales producciones que se obtienen de esta actividad se derivan del ganado bovino, porcino y avícola con $85 \%, 10.7 \%$ y $3.6 \%$, respectivamente (SIAP, 2012b).

\section{MATERIALES Y MÉTODOS}

El presente estudio está orientado a obtener la clasificación de uso de suelo y los cambios ocurridos a través del periodo 1994-2010. Estos cambios han sido estimados con la ayuda de dos imágenes Landsat Thematic Mapper (TM) obtenidas durante la estación de secas con una cobertura de nubosidad menor al 20\% (path/row: 25/46), técnicas de percepción remota, y el uso de Sistemas de Información Geográfica (SIG). Como resultado, se obtuvieron los mapas con las clases: selva, bosque, suelo agrícola, agua, vegetación perturbada, asentamientos urbanos, pastizales, cultivos de cítricos y matorrales para las fechas: 3 de diciembre de 1994 y 15 de diciembre de 2010.

\subsection{Pre-procesamiento de imágenes}

Las imágenes de satélite de las fechas seleccionadas se obtuvieron del portal de internet del Servicio Geológico de los Estados Unidos (United States Geological Survey - USGS). El motivo por el cual se seleccionaron estas fechas fue por la escasez de imágenes sin cobertura de nubosidad, problema recurrente en la zona de estudio. Las imágenes fueron georreferidas usando coordenadas en la proyección cartográfica Universal Transversal de Mercator (Zona 14 Norte), para su integración dentro del entorno SIG. Este proceso se llevó a cabo en el SIG Idrisi Selva (Eastman, 2010). Una vez que las imágenes tuvieron la misma georreferencia, se procedió a llevar a cabo las correcciones radiométricas y atmosféricas de cada imagen.

Las correcciones radiométricas son fundamentales para una adecuada interpretación de las imágenes satelitales, ya que los contrastes en la iluminación y la geometría deben ser minimizados en estudios multi-temporales mediante el uso de valores constantes o coeficientes de corrección de factores tales como la distancia entre la tierra y el sol (Alexakis et al., 2014; Chander et al., 2009; Schroeder et al., 2006). La corrección consistió en la utilización de ecuaciones estándar para convertir números digitales (ND) a unidades de radiancia y reflectancia percibida por el sensor del satélite. Las ecuaciones y los datos de calibración fueron obtenidos de Chander et al. (2009). Después de la conversión a radiancia, cada imagen fue convertida a reflectancia superficial considerando la corrección atmosférica. Este método asume que en una imagen de satélite existen superficies que reflejan una proporción de radiación solar muy baja (es decir, cuerpos de agua, sombras), de tal manera que la señal proveniente de estas superficies u objetos que es registrada por el sensor no representa una medida correcta (Chuvieco, 2010). Una alternativa para determinar la influencia atmosférica y reducir sus efectos dentro del proceso de calibración fue mediante la propia información de la imagen, para lo cual se empleó el método de substracción del objeto oscuro (DOS) propuesto por Chavez (1996).

\subsection{Clasificación de imágenes}

El procedimiento se realizó mediante una clasificación supervisada, utilizando el clasificador de máxima verosimilitud, ya que es uno de los algoritmos más comúnmente utilizados en percepción remota y es considerado como uno de los procedimientos de discriminación más eficientes, encontrándose estudios en México que lo han utilizado con resultados satisfactorios (Loya-Carrillo et al., 2013; Berlanga et al., 2010).

En el clasificador de máxima verosimilitud, el intérprete establece en la imagen campos de entrenamiento representativos de cada categoría de cobertura vegetal y uso de suelo, con base en los cuales se caracteriza estadísticamente cada categoría (fase de entrenamiento). 
Posteriormente, se evalúa una función de probabilidad multidimensional completa, en la que cada píxel es asignado a la clase de mayor probabilidad de pertenencia (Eastman, 2010). Es importante notar que este enfoque se basa únicamente en la información espectral de la imagen y no considera elementos como textura, forma y disposición de objetos (Berlanga et al., 2010).

En el presente estudio, los campos de entrenamiento fueron digitalizados de composiciones en falso color de las escenas, con el apoyo de las cartas de uso del suelo y vegetación de CONABIO e INEGI (series II y IV), y mediante la plataforma de Google Earth, con la intención de entrenar al programa con las clases a caracterizar. En promedio se seleccionaron alrededor de 30 campos de entrenamiento por clase, con 12 a 16 píxeles por campo para cada imagen, lo que representa áreas mayores a una hectárea. En este proceso se buscó que los píxeles de las áreas seleccionadas fueran homogéneos para cada categoría, haciendo coincidir los valores medios de los campos de entrenamiento pertenecientes a una misma clase, y discriminándolos del resto de las clases. Posteriormente se ejecutó el algoritmo de máxima verosimilitud en el software Idrisi Selva 17.0 (Eastman, 2010), mediante el cual, cada píxel fue agrupado de acuerdo a la probabilidad de que perteneciera a una clase dada (Eastman, 2003).

\subsection{Detección de cambios de cobertura vegetal y uso de suelo}

La evaluación del cambio de cobertura vegetal y uso del suelo en la cuenca del río Tecolutla se realizó a través de un enfoque de comparación post-clasificatorio utilizando como herramienta una matriz de transición. Este análisis permitió calcular los cambios observados en el periodo de estudio (1994-2010), al realizar una tabla con arreglos simétricos, conteniendo en uno de los ejes las clases de uso de suelo del primer año (1994) y en el otro eje las clases del segundo año (2010). De esta forma, los datos en la diagonal principal indican las áreas que han permanecido intactas en el tiempo para cada clase, mientras que aquéllos que se encuentran fuera, representan la transición de una clase a otra en forma de ganancia, si se encuentran en las filas, o pérdida si se encuentran en las columnas (Berlanga et al., 2010; López-Granados, 2001).

\subsection{Validación de resultados}

La muestra para realizar la comparación de resultados fue determinada mediante muestreo aleatorio estratificado. Un total de 384 píxeles fueron seleccionados para la validación, con un $95 \%$ de confianza. La validación fue realizada mediante una matriz de confusión, con la que se evaluó la exactitud de la clasificación, situando en las filas las clases o categorías obtenidas en el mapa y en las columnas las mismas clases con datos reales del terreno o campo. La precisión general se evaluó dividiendo el número de píxeles clasificados correctamente entre el total de lugares de referencia. Otra prueba estadística complementaria que se empleó, fue el coeficiente de Kappa, mediante el cual es posible reconocer los errores de comisión (precisión del usuario) y de omisión (precisión del productor). En los primeros se clasifican elementos que no pertenecen a una clase, mientras que en los segundos, no se clasifican elementos, por estar erróneamente incluidos en otra clase (Berlanga et al., 2010).

\section{RESULTADOS Y DISCUSIÓN}

A partir de la corrección radiométrica, geométrica y atmosférica de las imágenes de satélite Landsat 5 TM de 1994 y 2010 y su posterior clasificación supervisada con el algoritmo de máxima verosimilitud, se generaron dos mapas de uso de suelo, representando nueve usos y coberturas de suelo (selva, bosques, suelo agrícola, agua, vegetación perturbada, asentamientos urbanos, pastizales, cultivos de cítricos y matorrales). En las Figuras 2 y 3 se presentan las clasificaciones obtenidas, en base a las cuales, la mayor proporción de cambio 
se observa en el estado de Veracruz, donde se aprecia un cambio considerable de pastizales a zona agrícola. También se puede observar una disminución en la densidad de bosques, principalmente en el estado de Puebla.

Durante la validación, se evaluó la exactitud global para los años de 1994 y 2010, encontrándose exactitudes de $92.5 \%$ para el año de 1994 y de $93.1 \%$ para el 2010. Debido a que el algoritmo de máxima verosimilitud se basa únicamente en información espectral de cada píxel y no tiene capacidad para identificar formas, no fue posible diferenciar a los poblados de las zonas agrícolas, por lo que fue necesario incorporar los polígonos de estas clases, que fueron digitalizadas mediante la composición de imágenes, para ambos años, con la ventaja de hacerlo sin errores de omisión o comisión. Por otra parte, la clase de cultivos de cítricos en el año de 1994 presentó importantes errores de comisión, donde se clasificaron píxeles en coberturas a las que no pertenecían, confundiéndose con vegetación perturbada, suelo agrícola y bosque, lo cual probablemente se debió a los campos de entrenamiento seleccionados en ese año, puesto que no se contaba con una clasificación previa de esa clase.

En lo concerniente al índice de Kappa, se obtuvieron valores de 0.89 y 0.91 , para las clasificaciones de 1994 y 2010, respectivamente. De acuerdo a Viera y Garret (2005) al obtener un índice de Kappa mayor a 0.81 se considera una exactitud casi perfecta, por lo que las clasificaciones de los mapas pueden ser aceptadas como una buena aproximación a las condiciones reales. Esto permitió generar una figura que resume los cambios de uso de suelo que han ocurrido durante el periodo 1994-2010 (Figura 4).

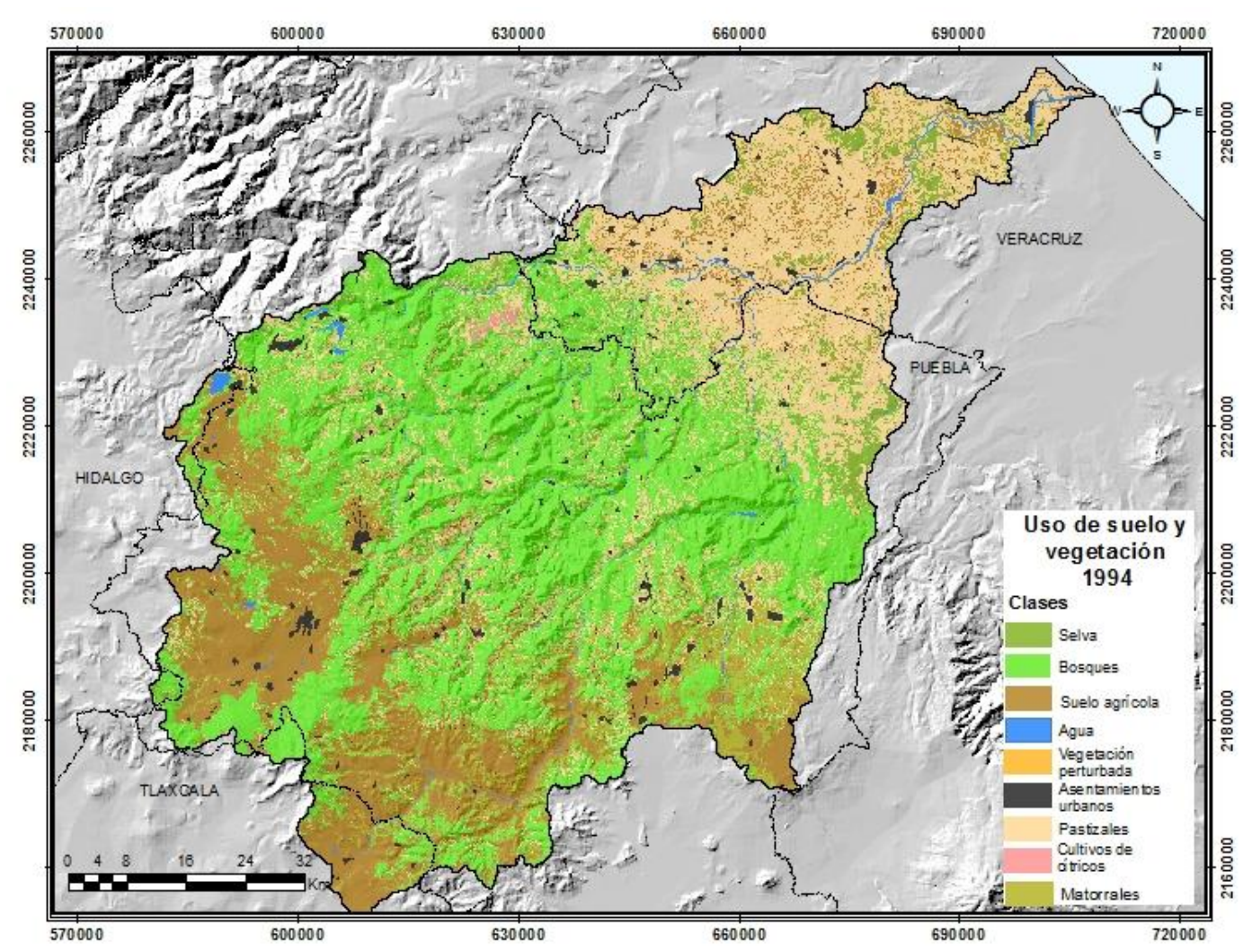

Figura 2. Uso de suelo y vegetación en 1994.

En el año de 1994 se presentó una mayor cobertura de bosques $(41.6 \%)$, seguida por pastizales $(24.8 \%)$, y finalmente por zona agrícola de temporal $(20.4 \%)$ y por debajo de estos porcentajes los otros usos. En el año 2010, predominaron los bosques (34.6\%), seguidos por zona agrícola de temporal $(26.2 \%)$, y por último pastizales $(25.8 \%)$, los otros usos se encuentran por debajo del $10 \%$. Por lo tanto, las áreas que mostraron mayores cambios con respecto a su extensión en 1994, fueron los asentamientos urbanos con 67.6\% (7,663 ha), 
vegetación perturbada con $41.2 \%$ (2,568 ha), selva con $33.3 \%$ (7,958 ha), cultivos de cítricos con $32.7 \%$ (642 ha), suelo agrícola con $28.2 \%$ (42,833 ha) y bosques con $16.9 \%$ (52,532 ha).

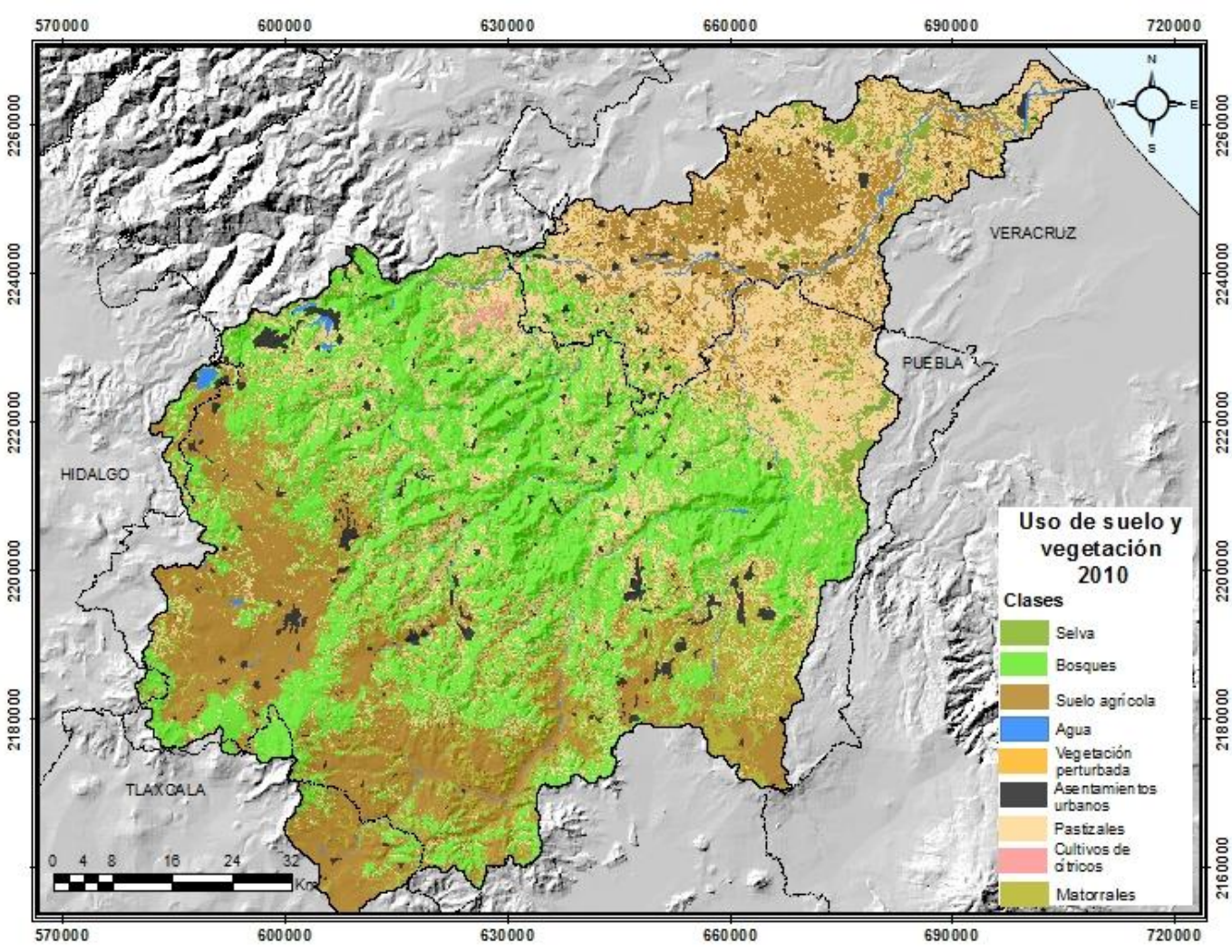

Figura 3. Uso de suelo y vegetación en 2010.

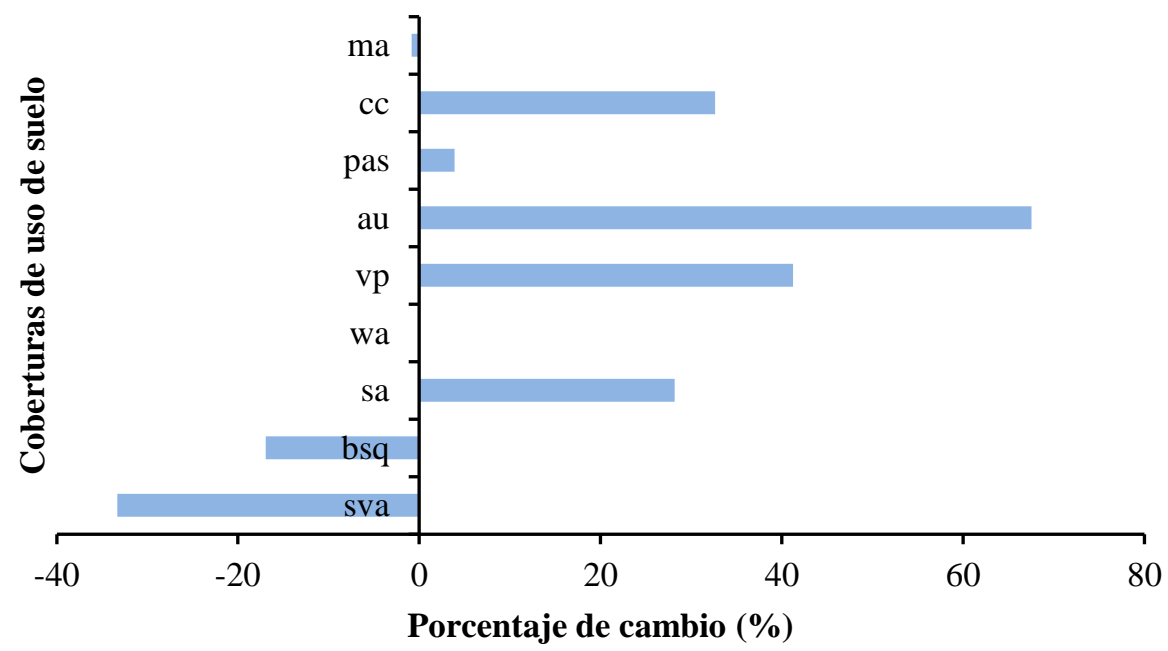

Figura 4. Cambios de uso de suelo en el periodo 1994-2010. Selva (sva), bosque (bsq), suelo agrícola (sa), agua (wa), vegetación perturbada (vp), asentamientos urbanos (au), pastizales (pas), cultivos de cítricos (cc), matorrales (ma). Valores negativos indican pérdidas y valores positivos representan ganancias.

En 1994 se contaba con una superficie forestal (bosques y selvas) de 333,866 ha, sin embargo, en 2010 esta cobertura disminuyó 60,490 ha, dando una tasa de deforestación media anual de $-1.1 \%$ la cual es mayor a lo reportado por la Organización de las Naciones Unidas 
para la Agricultura y Alimentación (FAO) (-0.52\%) en el periodo 1990-2010 en México (FAO, 2010). Por otra parte, en estudios realizados por Velázquez et al. (2002) y FAO (2001) para bosques y selvas, se reporta una tasa de deforestación de $-1.1 \%$ en México, para los periodos 1993-2000, y 1990-2000, respectivamente, lo cual se asemeja a lo presentado en el presente estudio.

En el análisis de los patrones de cambio, se generó la matriz de transición que explica claramente los cambios ocurridos (Tabla 1), donde las filas y columnas representan las pérdidas y ganancias (en porcentaje) que se han producido por cambios de cobertura y uso del suelo, así como también las áreas que han permanecido intactas hasta el 2010 (diagonal principal). También se construyó un mapa con las pérdidas más representativas de cobertura y uso de suelo en el periodo 1994-2010 (Figura 5).

En los 16 años de estudio (1994-2010), las actividades agropecuarias (suelo agrícola y pastizales) han influido en mayor medida a propiciar la disminución de cobertura vegetal en la cuenca, ya que se utilizaron 6,206 ha de selva (26\%) y 46,774 ha de bosque (15.1\%) para el desarrollo de estas actividades. Por otra parte, también se observó una importante transición de selva y bosque a vegetación perturbada, con un $8 \%$ y $0.8 \%$, respectivamente $(1,907$ y $2567 \mathrm{ha}$ ) en la cuenca, reflejando el proceso de transformación de uso de suelo, donde primero se observa una transición de coberturas naturales (bosques y selvas) a vegetación perturbada y posteriormente esta es transformada a usos agropecuarios, donde en este estudio se obtuvo una transformación de $24.9 \%$ a suelo agrícola y 14.7 a pastizales. La zona de la cuenca en donde se pueden apreciar mayores pérdidas de cobertura de bosque y selva es principalmente en el límite fronterizo entre los estados de Veracruz y Puebla. Asimismo, las áreas naturales con mayor conservación se concentran en sitios con terrenos muy accidentados y con pendientes pronunciadas, como se puede observar en el estado de Puebla.

Por otra parte, también se ha observado una importante transición de pastizales a zonas agrícolas con 32,646 ha (17.7\%) en el estado de Veracruz, reflejando el importante crecimiento que ha tenido esta actividad en la región.

Tabla 1. Matriz de transición de cambio de uso del suelo entre 1994-2010 (\% de hectáreas).

\begin{tabular}{lcccccccc}
\hline $\begin{array}{l}\text { Uso del } \\
\text { suelo }\end{array}$ & sva & bsq & sa & vp & au & pas & cc & ma \\
\hline sva & $\mathbf{6 5 . 6}$ & 0.0 & 6.5 & 8.0 & 0.2 & 19.5 & 0.3 & 0.0 \\
bsq & 0.0 & $\mathbf{8 3 . 0}$ & 3.0 & 0.8 & 0.8 & 12.1 & 0.2 & 0.0 \\
sa & 0.1 & 0.0 & $\mathbf{9 8 . 2}$ & 0.1 & 1.0 & 0.5 & 0.0 & 0.1 \\
vp & 0.3 & 0.0 & 24.9 & $\mathbf{5 9 . 3}$ & 0.8 & 14.7 & 0.0 & 0.0 \\
au & 0.0 & 0.0 & 0.0 & 0.0 & $\mathbf{1 0 0 . 0}$ & 0.0 & 0.0 & 0.0 \\
pas & 0.0 & 0.0 & 17.7 & 0.3 & 1.8 & $\mathbf{8 0 . 0}$ & 0.1 & 0.0 \\
cc & 0.3 & 0.2 & 12.5 & 0.2 & 0.4 & 7.4 & $\mathbf{7 9 . 1}$ & 0.0 \\
ma & 0.0 & 0.0 & 0.6 & 0.0 & 0.5 & 0.3 & 0.0 & $\mathbf{9 8 . 6}$ \\
\hline
\end{tabular}

Selva (sva), bosque (bsq), suelo agrícola (sa), vegetación perturbada (vp), asentamientos urbanos (au), pastizales (pas), cultivos de cítricos (cc), matorrales (ma).

Por otra parte, también se observaron cambios inverosímiles en la matriz de transición, como el paso de suelo agrícola a selva con $0.1 \%$ (104 ha), de pastizales y bosques a selvas con $0.05 \%$ y $0.01 \%$, respectivamente ( 88 ha y 21 ha), y de cultivos de cítricos a selva y bosque con $0.3 \%$ y $0.2 \%$, respectivamente ( 5 ha y 4 ha). Esto se debe, a que éste método no presenta una exactitud del $100 \%$ y con éste análisis se transponen los errores individuales de cada uno de los mapas, desencadenando la propagación multiplicativa del error. Sin embargo, las hectáreas mal clasificadas son bajas en comparación con el número de hectáreas 
clasificadas y los niveles de exactitud son altos (mayores a 92\%), por lo que los resultados obtenidos con esta técnica pueden considerarse como satisfactorios (Berlanga et al., 2010).

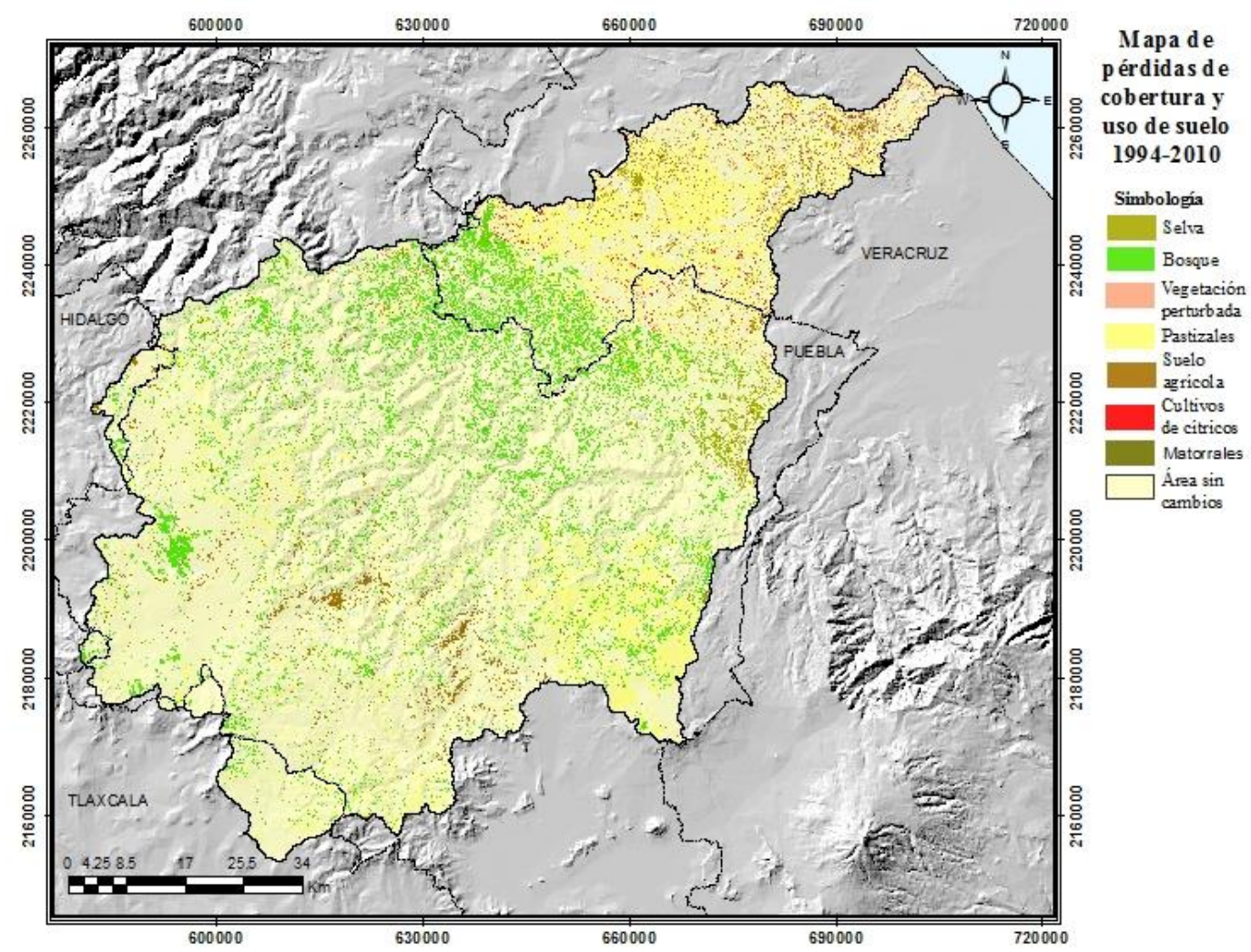

Figura 5. Mapa de pérdidas de coberturas y uso de suelo en el periodo 1994-2010.

El presente estudio ofrece una caracterización del cambio de uso de suelo en la cuenca del río Tecolutla durante el periodo 1994-2010, mediante el uso del procedimiento de máxima verosimilitud. Nuestros resultados vienen a corroborar estimaciones sobre la proporción de superficies de ciertas coberturas y sus cambios, expuestas en otros trabajos de investigación dentro del estado de Veracruz, tal es el caso del trabajo de Toledo y Ordoñez (1998), quienes encontraron que en el trópico húmedo veracruzano el 19\% de la selva ha sido convertida a pastizales, mientras que en este estudio el resultado fue de 19.5\%. También existió coincidencia con los resultados de Hernández et al. (2013), quienes determinaron que las principales causas de deforestación en las selvas ubicadas en el sur del estado han sido provocadas por el crecimiento de la actividad agropecuaria a costa de la cobertura vegetal existente.

Trabajos como el de Ellis et al. (2011) exhiben la presencia de focos rojos para la conservación de la biodiversidad, quienes han caracterizado la cobertura de vegetación y uso de suelo en Veracruz, basado en percepción remota. El estudio muestra los efectos del uso ganadero y la concentración de usos agrícolas sobre la vegetación natural de ciertas regiones del estado (Áreas Naturales Protegidas- ANP). En este sentido, existe una coincidencia con los resultados obtenidos en este trabajo, donde se observa que los sitios más afectados han sido propiciados por la expansión agropecuaria, incidiendo principalmente en la cobertura de selvas, bosques y pastizales. 


\section{CONCLUSIONES}

La integración de los SIG y la teledetección proporcionó información valiosa sobre la magnitud de los cambios asociados al crecimiento de las actividades antropogénicas a costa de la vegetación natural existente, durante el periodo 1994-2010.

Dentro de las actividades desarrolladas en la cuenca del río Tecolutla, las actividades agrícolas han jugado un rol preponderante en los últimos años, presentando un aumento considerable (incremento de 74\%), principalmente en el estado de Veracruz. Dicho suceso es entendible desde el punto de vista socioeconómico, ya que dichas actividades representan una fuente de ingreso a corto plazo para los habitantes. Sin embargo, la intensidad de estas actividades ha provocado una pérdida de cobertura natural (selvas y bosques), lo cual puede traer consecuencias en la fertilidad y erosión del suelo. Por otra parte, también se observó una importante transición de selvas y bosques a vegetación perturbada, y esta a su vez en pastizales y suelo agrícola, reflejando la sucesión ecológica que experimenta la vegetación natural en la cuenca.

Esta información es importante, ya que puede apoyar en la elaboración de planes de ordenamiento territorial, en la planeación de estrategias de conservación de los recursos naturales en la cuenca y en la búsqueda de patrones óptimos de uso de suelo en los municipios y localidades ubicados en la cuenca del río Tecolutla, Veracruz.

\section{AGRADECIMIENTOS}

Al Fondo Mixto CONACYT-Gobierno del Estado de Veracruz, por el financiamiento al proyecto VER-2008-C01-96299 que permitió la realización de este trabajo. Ana Karen Osuna-Osuna agradece al CONACYT la beca de Maestría con número de registro 301696.

\section{REFERENCIAS}

ALEXAKIS, D. D.; GRILlAKIS, M. G.; KOUTROULIS, A. G.; AGAPIOU, A.; THEMISTOCLEOUS, K.; TSANIS, I. K. et al. GIS and remote sensing techniques for the assessment of land use change impact on flood hydrology: the case study of Yialias basin in Cyprus. Natural Hazards and Earth System Science, v. 14, n. 2, p. 413-426, 2014. http://dx.doi.org/10.5194/nhess-14-413-2014

BERlangA, C. A.; GARCÍA, R. R.; LÓPEZ, J.; RUÍZ, A. Patrones de cambio de coberturas y usos del suelo en la región costa norte de Nayarit (1973-2000). Investigaciones Geográficas, n. 72, p. 7-22, 2010.

CHANDER, G.; MARKHAM, B. L.; HELDER, D. L. Summary of current radiometric calibration coefficients for Landsat MSS, TM, ETM+, and EO-1 ALI sensors. Remote Sensing of Environment, v. 113, n. 5, p. 893-903, 2009. http://dx.doi.org/10.1016/j.rse.2009.01.007

CHAVEZ, P. S. Image-Based Atmospheric Corrections - Revisited and Improved. Engineering and Remote Sensing, v. 62, n. 9, p. 1025-1036, 1996.

CHUVIECO, E. Teledetección ambiental. La observación de la Tierra desde el espacio. España: Ariel, 2010. 528 p.

COMISIÓN NACIONAL DEL AGUA - CONAGUA (México). Atlas del agua en México, 2012. México: SEMARNAT, 2012. 133 p. 
DZIESZKO, P. Land-cover modelling using corine land cover data and multi-layer perceptron. Quaestiones Geographicae, v. 33, n. 1, p. 5-22, 2014. http://dx.doi.org/10.2478/quageo-2014-0004

EASTMAN, J. R. IDRISI Kilimanjaro: guide to GIS and image processing. Worcester: Clark Labs, Clark University, 2003. 306 p.

EASTMAN, J. R. IDRISI Selva, version 17.0. Worcester: Clark University, 2010.

ELLIS, E. A.; MARTÍNEZ-BELLO, M.; MONROY-IBARRA, R. Focos rojos para la conservación de la biodiversidad en el estado de Veracruz. In: CRUZ-ANGÓN, A. (Ed.). La biodiversidad en Veracruz: estudio de Estado. México: CONABIO; Gobierno del Estado de Veracruz; Universidad Veracruzana; Instituto de Ecología, 2011. p. 351-368.

FOOD AND AGRICULTURE ORGANIZATION OF THE UNITED NATIONS - FAO. FRA2000-Causas y tendencias de la deforestación en América Latina. Roma, 2001.

FOOD AND AGRICULTURE ORGANIZATION OF THE UNITED NATIONS - FAO. Global Forest Resources Assessment. Roma, 2010.

HERNÁNDEZ, I. U.; ELLIS, E. A.; GALLO, C. A. Aplicación de teledetección y sistemas de información geográfica para el análisis de deforestación y deterioro de selvas tropicales en la región Uxpanapa, Veracruz. GeoFocus, n. 13, p. 1-24, 2013.

IBARRA-MONTOYA, J. L.; ROMÁN, R.; GUTIÉRREZ, K.; GAXIOLA, J.; ARIAS, V.; BAUTISTA, M. Cambio en la cobertura y uso de suelo en el norte de Jalisco, México: Un análisis del futuro, en un contexto de cambio climático. Revista Ambient. Agua, Taubaté, v. 6, n.2, p. 111-128, 2011. http://dx.doi.org/10.4136/ambi-agua.189

INSTITUTO NACIONAL DE GEOGRAFÍA, ESTADÍSTICA E INFORMÁTICA - INEGI. Síntesis de información geográfica del estado de veracruz. Aguascalientes, 2005.

INSTITUTO NACIONAL DE GEOGRAFÍA, ESTADÍSTICA E INFORMÁTICA - INEGI. Carta de uso de suelo y vegetación Serie IV, escala 1:250 000. Aguascalientes, 2007.

LONG, H.; HEILIG, G. K.; LI, X.; ZHANG, M. Socio-economic development and land-use change: Analysis of rural housing land transition in the Transect of the Yangtse River, $\begin{array}{llllll}\text { China. Land Use Policy, v. 24, p. 141-153, } 2007 . & \end{array}$ http://dx.doi.org/10.1016/j.landusepol.2005.11.003

LÓPEZ-GRANADOS, E. M.; BOCCO, G.; MENDOZA, M. E. Predicción del cambio de cobertura y uso del suelo. El caso de la ciudad de Morelia. Investigaciones Geográficas, n. 45, p. 56-76, 2001.

LOYA-CARRILlO, J. O.; AGUILAR-ESTRADA， S. A.; BRAVO-PEÑA， L. C.; SÁNCHEZ-FLORES, E. Evaluación espacio-temporal del impacto del crecimiento urbano sobre la cobertura vegetal en la región fronteriza Nogales, México y Arizona, Estados Unidos, durante el periodo 1995-2010. Revista Latinoamericana de Recursos Naturales, v.9, n. 1, p. 124-140, 2013.

PÉREZ-SESMA, J. A. A. Estudio del agua de escurrimiento en la cuenca del río Tecolutla, México. 2009. Tesis (Maestría en Geografía) - Facultad de Filosofía y Letras, UNAM, México. 2009. 
PÉREZ-MAQUEO, O.; MUÑOZ-VILLERS, L.; VÁZQUEZ, G.; EQUIHUA, M.; LEÓN, P. Amenazas a los recursos hídricos. In: COMISIÓN NACIONAL PARA EL CONOCIMIENTO Y USO DE LA BIODIVERSIDAD - CONABIO (Ed.). La biodiversidad en Veracruz: estudio de estado. México: CONABIO; Gobierno del Estado de Veracruz; Universidad Veracruzana; Instituto de Ecología, 2011. p. 327-338.

RODRÍGUEZ-GALIANO, V. F.; GARCÍA-SOLDADO, M. J.; CHICA-OLMO, M.; PARDO-IGÚZQUIZA, E.; RIGOL-SÁNCHEZ, J. P.; CHICA-RIVAS, M. Análisis de cambios de usos del suelo en la "Vega de Granada": correcciones radiométricas y evaluación del cambio. Revista de Teledetección, v. 34, p. 5-15, 2010.

SCHROEDER, T. A.; COHEN, W. B.; SONG, C.; CANTY, M. J.; YANG, Z. Radiometric correction of multi-temporal Landsat data for characterization of early successional forest patterns in western Oregon. Remote Sensing of Environment, v. 103, n. 1, p. 16-26, 2006. http://dx.doi.org/10.1016/j.rse.2006.03.008

SERVICIO DE INFORMACIÓN ESTADÍSTICA AGROPECUARIA Y PESQUERA SIAP. Cierre de la producción agrícola por estado. 2012a. Disponible en: http://www.siap.gob.mx. Acceso: Diciembre 2013.

SERVICIO DE INFORMACIÓN ESTADÍSTICA AGROPECUARIA Y PESQUERA SIAP. Resumen municipal pecuario. 2012b. Disponible en: http://www.siap.gob.mx. Acceso: Diciembre 2013.

TOLEDO, V. M.; ORDÓÑEZ, M. J. El panorama de la biodiversidad de México: una revisión de los hábitats terrestres. In: RAMAMOORTHY, T. P.; BYE, R.; LOT, A.; FA, Y. A. (Eds.). Diversidad biológica de México. México: Instituto de Biología, UNAM, 1998. p. 739-757.

TREJO, I. Enriqueta García Amaro de Miranda (Una investigadora incansable). Investigaciones Geográficas, n. 41, p. 175-176, 2000.

VELÁZQUEZ, A.; MAS, J. F.; DÍAZ, G. J.; MAYORGA, S. R.; ALCÁNTARA, P. C.; CASTRO, R. et al. Patrones y tasas de cambio de uso del suelo en México. Gaceta Ecológica, n. 62, p. 21-37, 2002.

VIERA, A. J.; GARRETT, J. M. Understanding interobserver agreement: the kappa statistic. Family medicine, v. 37, n. 5, p. 360-363, 2005. 